\title{
Knowledge, attitude, and practices of the healthcare workers regarding 2019 novel coronavirus disease - A questionnaire study
}

\author{
Ashna Bhasin ${ }^{1}$, Poonam Loomba ${ }^{2}$, Abha Sharma ${ }^{3}$, Bibhabati Mishra ${ }^{4}$, Sulmaz Reshi ${ }^{1}$

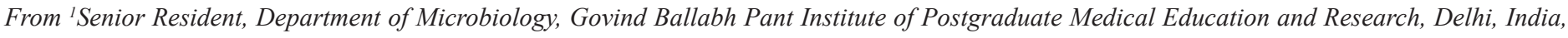 \\ ${ }^{2}$ Director Professor and HOD, Department of Microbiology, Govind Ballabh Pant Institute of Postgraduate Medical Education and Research, Delhi, \\ India, ${ }^{3}$ Associate Professor, Department of Microbiology, Govind Ballabh Pant Institute of Postgraduate Medical Education and Research, Delhi, \\ India, ${ }^{4}$ Consultant, Department of Microbiology, Govind Ballabh Pant Institute of Postgraduate Medical Education and Research, Delhi, India
} Correspondence to: Dr. Ashna Bhasin, IA-15 B, Ashok Vihar, Phase-1, Delhi - 110 052, India. E-mail: bhasinashna@gmail.com Received - 15 December 2020

Initial Review - 26 December 2020

Accepted - 13 January 2021

\section{ABSTRACT}

Background and Objective: The 2019 novel coronavirus disease (COVID-19) pandemic in India has affected over 8.14 million people and is a major concern for the people and governments across due to its impact on individuals as well as on public health. The present study striven to determine knowledge, attitude, and practices of healthcare workers (HCWs) toward COVID-19 in a super specialty center. Materials and Methods: A questionnaire-based survey was carried out among $100 \mathrm{HCWs}$ belonging to the different cadre and specialties of a Super specialty hospital in North India. Questionnaire focused on the awareness, attitude, and possible practices toward ensuring safety for themselves as well as breaking the chain of transmission. A convenient sampling method was used for data collection and descriptive statistical analysis was carried out to assess the data. Results: Of the 100 participants, 52 were male and 48 were female mostly belonging in the age group of 25-40 years. Most participants (80\%) used social media to obtain information on COVID-19. More than $80 \%$ of the respondents knew and practiced different precautionary measures. However, about 31\% cases were associated with poor attitude and practices of COVID-19. Conclusion: Most HCWs surveyed were well informed about novel severe acute respiratory syndrome coronavirus 2 and took adequate measures in preventing the spread of infection. However, continued professional education is advised among HCWs to curtail the spread of this disease.

Key words: 2019 novel coronavirus disease, Healthcare workers, Knowledge, attitude, and practices

$\mathrm{T}$ he 2019 novel coronavirus disease (COVID-19) caused by novel severe acute respiratory syndrome coronavirus 2 (SARS-CoV-2) emerged in Hubei province of China in the winter months, followed by its rapid spread all over the world. On March 11, 2020, the World Health Organization (WHO) declared it as a pandemic. Despite lockdown, COVID-19 had caused over thousands of deaths in countries across the world and there is no end to this ordeal. COVID-19 is unfold by human-to-human transmission through droplet, feco-oral, direct contact and has an incubation period of 2-14 days. Till date, no antiviral treatment or vaccine has been explicitly recommended for COVID-19. Therefore, applying preventive measures to control COVID-19 infection is the most critical intervention [1]. Healthcare workers (HCWs) play a central role in providing quality healthcare and preventing the spread of COVID-19 by breaking the chain. However, they themselves are at maximum risk of getting infected. Therefore, a wide range of health-care services must be ensured for effective prevention of COVID-19 infection spread [2].

Despite the risk of infection for this group is high, there is also a risk of stigma due to perceptions that HCWs have high risk of spreading the infection [3]. Research conducted during the previous epidemics have helped to identify how organizational and social factors, including HCW confidence in ability to effectively deliver infection prevention and control measures, were important to protect both physical and psychological health [4]. Pointers for HCWs and on-line refresher courses have been developed by WHO, CDC, and various governmental organizations in various countries to spice up the information and prevention strategies [5]. However, there is a lot of gap between the exact knowledge and how the different HCWs have perceived it. Hence, it is crucial to understand HCWs risk perception and to identify their trusted sources of information to effectively communicate and frame key messages in response to the emerging disease. Hence, the study was carried out with an objective to assess the knowledge, attitude and practices of HCWs in a tertiary care super specialty non-COVID hospital.

\section{MATERIALS AND METHODS}

This cross-sectional study was conducted in the Department of Microbiology, Govind Ballabh Pant Institute of Postgraduate Medical Education and Research, New Delhi, India, from July 2020 to August 2020 during the COVID-19 unlock period. The 
study was approved by the Institutional Ethics Committee (Ref No-2020/10/06-03). A total of 100 participants were above 18 years of age including consultants, residents, interns, medical students, physiotherapists, physiotherapy students, nurses, nursing students, and technicians working in our hospital which were selected through convenience sampling.

The survey was divided into three parts, including participant information sheet, informed consent form, and questionnaire. An informed consent was obtained from all participants. Selfadministered printed questionnaire was in English language comprising of closed-ended and open-ended questions with completion time of 5-10 min. Demographics included sex, age, academic qualification, highest level of education, and sources of information on COVID-19. The questionnaire consisted of 40 questions including general knowledge about novel Coronavirus (22 items), attitude (4 item), and practices of COVID-19 (14 item-9 open-ended and five closed-ended questions).

The questions on knowledge were comprised two item questions based on the medical presentations, transmission, prevention, and manipulate of COVID-19. Each accurate response was scored 1 point and zero for wrong responses. A total score of less than 10 points accounted for poor knowledge, score between 10 and 16 accounted for average knowledge and a score above 17 meant that the HCW has good knowledge about COVID-19 [6]. The responses to the questions in attitude category were strongly disagree, disagree, neutral, agree and strongly agree, weighing 1-five, respectively, for every superb statement. Some questions were reversed to put off biases of giving comparable reaction in all of the items. A score of less than 10 accounted for poor attitude, 10-15 accounted for average attitude and score of more than 15 points meant that the HCW had a good attitude toward COVID-19 [7].

Practices were assessed using five Likert-item questions that have been developed from the WHO recommended practices for prevention of COVID- 19 transmission, that is, hand washing, avoiding crowded places, keeping social distance (1 m apart), avoiding touching of face, and avoiding handshakes. The responses options were always, occasional, and by no means every weighing 3,2, and 1 factors, respectively. A total score of less than 11 points suggested poor practices and more than 12 points score suggested good practices to combat COVID-19. In addition, practices were also assessed by asking nine open-ended questions. The obtained data were analyzed using descriptive statistics. Due to large sample size in the HCWs, exploratory visual comparisons were presented without typical statistical tests of significance.

\section{RESULTS}

A total of 100 health and allied professionals consented and completed the survey. Table 1 summarizes the demographic data of the participants. The respondents of the study belonged to the age group of 25-40 years with equal gender distribution. Majority of the $\mathrm{HCW}$ who responded to the survey were technicians (31\%) and doctors $(30 \%)$. Of the 100 participants, at least $64 \%$ had bachelor's degree and a minority had an ordinary diploma or a certificate. The chief fountain of facts about COVID-19 among participants was information from social media $(80 \%)$ such as WhatsApp and Facebook. Figure 1 shows the percentage of the sources of information of the HCWs.

Majority of the HCWs had good or sufficient knowledge (80\%) on COVID 19; while, poor knowledge was found only in $2 \%$ of the HCWs (Fig. 2). Most health-care professionals (88\%) correctly identified the main symptoms of SARS-CoV-2 infection. Respondents were aware of precautionary measures such as hand washing/use of hand sanitizer, wearing masks, social distancing, covering mouth while sneezing, and self-quarantine (100\%). Majority of the participants were aware of the infective period and the asymptomatic period (100\%). Most participants rightly endorsed medical masks for HCWs, symptomatic patients, and persons who are coughing/sneezing. However, an appreciable proportion of health-care professionals (28\%), wrongly endorsed medical masks for healthy persons to protect themselves. Responses to knowledge based questions are summarized in Table 2.

Most of the participants in our study were worried about getting SARS-CoV-2 infection. Those who were not worried expressed justified reasons (mainly precautions) for their attitude.

Table 1: Socio-demographic characteristics of healthcare workers

\begin{tabular}{lc}
\hline Variables & Frequency \\
\hline Sex & 52 \\
Male & 48 \\
Female & \\
Healthcare worker category & 18 \\
Nurse & 31 \\
Doctor & 9 \\
NO & 12 \\
Housekeeping & 30 \\
Technician & \\
Highest level of education & 35 \\
Diploma & 5 \\
Certificate & 21 \\
Masters & 29 \\
Bachelors & 2 \\
Uneducated & 8 \\
High school & \\
\hline
\end{tabular}

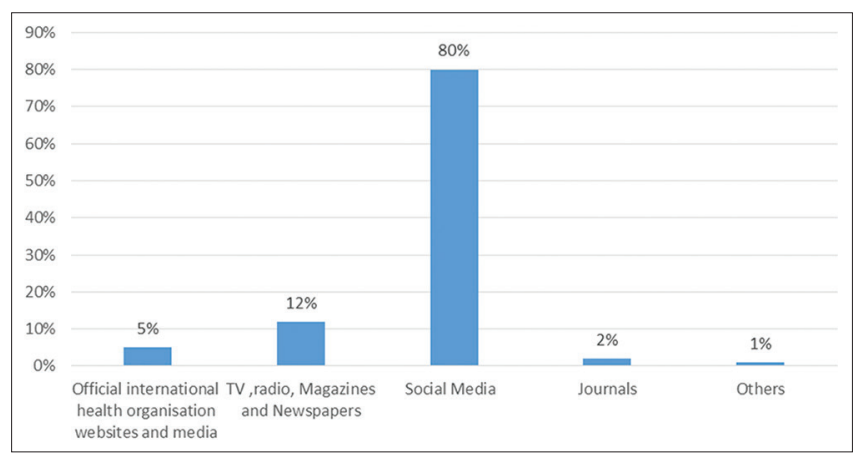

Figure 1: Sources of information on 2019 novel coronavirus disease 
Sixty-six percent HCWs had good attitude while $15 \%$ and $19 \%$ had average and poor attitude respectively towards COVID-19 (Table 3 and Fig. 2). Most health-care professionals expressed their distrust in the government task force on containing SARS-CoV-2 infection. Sixty-nine percent health care workers (HCWs) had good practices in preventing COVID-19 infection (Table 4 and Fig. 2). About $97 \%$ of the HCWs wore a mask while in contact with patient and washed their hands before and after touching the patient. In our study, $43 \%$ HCWs knew the proper donning and doffing techniques of PPE while, 58\% HCWs knew how to correctly discard gloves,

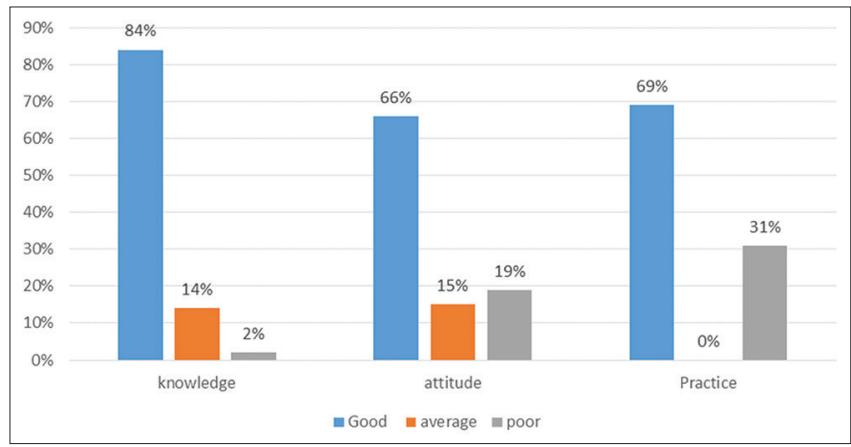

Figure 2: Overall knowledge, attitude, and practice scores of health care workers about 2019 novel coronavirus disease masks, and PPE. Only 34\% knew how to check whether N95 masks have been worn correctly while $56 \%$ were aware of the correct chemical used for disinfection of COVID-19 area.

\section{DISCUSSION}

The COVID-19 pandemic, which was initially reported in the Wuhan city of China, has raised serious concerns about the outbreak readiness across the world, including in the developed nations with the best quality health-care delivery system. The initial facts depict that the primary mode of disease transmission is airborne; there is an indispensable need to revisit the infection control practices. Apart from the various strategies proposed to strengthen the level of preparedness of a nation to contain the infection, proper training of the HCWs is also paramount. HCWs are the forefront warriors against the highly contagious disease and are always at a greater risk of acquiring the infection than the general public. Henceforth, it is of utmost importance that the HCWs should have proper and adequate knowledge regarding clinical features, diagnosis, treatment, and preventive measures to curtail the rapid spread of the disease. The present study evaluated the awareness of SARS-CoV-2 among health-care professionals

Table 2: Knowledge of the Healthcare workers toward COVID-19

\begin{tabular}{|c|c|c|c|c|}
\hline S. No. & The main clinical symptoms of COVID-19 are & $\begin{array}{l}\text { True } \\
(\%)\end{array}$ & $\begin{array}{c}\text { False } \\
(\%)\end{array}$ & $\begin{array}{l}\text { Don't know } \\
(\%)\end{array}$ \\
\hline 1 & Fever (True) & 100 & 0 & 0 \\
\hline 2 & Headache (True) & 100 & 0 & 0 \\
\hline 3 & Myalgia (True) & 95 & 0 & 5 \\
\hline 4 & Smell disturbance (True) & 80 & 5 & 15 \\
\hline 5 & Sneezing (True) & 80 & 2 & 18 \\
\hline 6 & Diarrhea (True) & 81 & 19 & 0 \\
\hline 7 & Cough (True) & 99 & 0 & 1 \\
\hline 8 & Confusion (True) & 72 & 3 & 25 \\
\hline 9 & $\begin{array}{l}\text { There is currently no effective cure for COVID-19, but early symptomatic and supportive treatment can } \\
\text { help most patients recover from the infection (True) }\end{array}$ & 93 & 2 & 5 \\
\hline 10 & $\begin{array}{l}\text { Not all persons with COVID-19 will develop severe cases, Only those who are elderly, have chronic } \\
\text { illnesses, and are obese are more likely to be severe cases (True) }\end{array}$ & 86 & 2 & 12 \\
\hline 11 & Eating or contacting wild animals would result in the infection by the COVID-19 virus (False) & 2 & 87 & 11 \\
\hline 12 & Persons with COVID-2019 cannot transmit the virus to others when a fever is not present (False) & 15 & 85 & 0 \\
\hline 13 & The COVID-19 virus spreads via respiratory droplets of infected individuals (True) & 97 & 2 & 1 \\
\hline 14 & Wearing a mask can prevent COVID-19 infections (True) & 100 & 0 & 0 \\
\hline 15 & $\begin{array}{l}\text { It is not necessary for children and young adults to take measures to prevent the infection by the } \\
\text { COVID-19 virus (False) }\end{array}$ & 0 & 100 & 0 \\
\hline 16 & $\begin{array}{l}\text { To prevent the infection by COVID-19, individuals should avoid going to crowded places such as bus } \\
\text { parks and avoid taking public transportations (True) }\end{array}$ & 85 & 0 & 15 \\
\hline 17 & Isolation and treatment of COVID-19 patients are effective ways to reduce the spread (True) & 100 & 0 & 0 \\
\hline 18 & $\begin{array}{l}\text { People who have contact with someone infected with the COVID-19 virus should be immediately isolated } \\
\text { in a proper place. In general, the observation period is } 14 \text { days (True) }\end{array}$ & 100 & 0 & 0 \\
\hline \multirow[t]{5}{*}{19} & How much contact is required to contract infection while in contact with a COVID-19 patient? & & & \\
\hline & While crossing the patient (False) & 90 & 7 & 3 \\
\hline & Sitting in the same room with mask (False) & 1 & 98 & 1 \\
\hline & Sitting in the same room without mask (True) & 100 & 0 & 0 \\
\hline & Having tea without one arm distance (True) & 82 & 5 & 13 \\
\hline
\end{tabular}


Table 3: Attitude of the Healthcare workers toward COVID-19

\begin{tabular}{|c|c|c|c|c|c|c|}
\hline S. No. & Questions & $\begin{array}{l}\text { Strongly disagree } \\
\text { (1 point) }\end{array}$ & $\begin{array}{l}\text { Disagree } \\
\text { (2 point) }\end{array}$ & $\begin{array}{l}\text { Not sure } \\
\text { ( } 3 \text { point) }\end{array}$ & $\begin{array}{c}\text { Agree } \\
\text { (4 point) }\end{array}$ & $\begin{array}{l}\text { Strongly agree } \\
\text { (5 point) }\end{array}$ \\
\hline 1 & $\begin{array}{l}\text { Wearing a well-fitting face mask is effective in preventing } \\
\text { COVID-19 }\end{array}$ & - & - & - & 2 & 98 \\
\hline 2 & $\begin{array}{l}\text { Using a hand wash can prevent you from getting } \\
\text { COVID-19 }\end{array}$ & 2 & 3 & 5 & 10 & 85 \\
\hline 3 & $\begin{array}{l}\text { When a patient has signs and symptoms of COVID-19, I } \\
\text { can confidently participate in the management of the patient }\end{array}$ & - & - & 2 & 15 & 83 \\
\hline 4 & India is in a good position to contain COVID-19 & 74 & 1 & 25 & - & - \\
\hline
\end{tabular}

Table 4: Practices of the Healthcare workers toward COVID-19

\begin{tabular}{|c|c|c|c|c|}
\hline S. No. & Questions & $\begin{array}{c}\text { Always } \\
\text { (1 point) }\end{array}$ & $\begin{array}{l}\text { Occasional } \\
\text { (2 points) }\end{array}$ & $\begin{array}{c}\text { Never } \\
(3 \text { points })\end{array}$ \\
\hline 1 & In recent days, I have gone to any crowded place? & 3 & 55 & 42 \\
\hline 2 & In recent days, I have worn a mask when in contact with patients? & 97 & 2 & 1 \\
\hline 3 & In recent days, I have refrained from shaking hands? & 100 & - & - \\
\hline 4 & In the recent days, I have washed my hands before and after handling each patient? & 97 & 3 & - \\
\hline 5 & In the recent days, I have avoided patients with signs and symptoms suggestive of COVID-19 & 48 & 51 & 1 \\
\hline
\end{tabular}

in a Super specialty Hospital during the unlock phase in rapidly developing country of Southeast Asia.

It is heartening to note that the knowledge with respect to SARS-CoV-2 was relatively high among the respondents $(80 \%)$. Similar findings were observed by studies done from the US and China [8]. Our study was in accordance to a study by Bhagavathula et al. who reported that the participants' main source of information was official government websites $(33 \%)$ followed by social media (30\%) [1]. Another survey conducted in Pakistan reported that the Social Media (87.68\%) remained the primary source of information among health-care professionals [9]. Increased knowledge observed in our study could be attributed to the fact that HCWs had been trained by the various workshops conducted in our hospital during the lockdown phase. In addition, increased use of social media and active media through television during lockdown could have enhanced knowledge. Hence, it is essential to ensure that accurate information through verified channels and health-care professionals are presented and broadcasted to the people.

This also points toward the importance of the right people being active on social media so that they can communicate the scientifically validated information to the masses. At present, there is a vast diversity of information available through the internet, including unverified malicious information that can spread quickly and misguide HCWs. In particular, the government and scientists have warned that huge incorrect information approximately COVID-19 is a critical problem inflicting xenophobia worldwide [10]. In this regard, HCWs ought to cautiously examine COVID-19-associated data, use clinical, and genuine content material as data sources. In a pandemic situation, this disparity in access can be catastrophic and hence most journals have provided open access to all coronavirus-related publications. Health-care professionals accessed websites such as WHO, CDC, and ICMR for reliable information, which is an indicator of their faith in health organizations across the world. In addition, online webinars through zoom or other applications conducted during the pandemic positively affected the responses of our study.

The severity of illness was well identified by all who were surveyed as being difficulty in breathing. Another heartening aspect was that precautionary measures were well known to participants with appropriate hand washing techniques, avoidance of public gatherings, and covering of the mouth while coughing and sneezing as the top three precautionary measures. There was less knowledge related to treatment and vaccine among both health-care professionals, which was a disappointing finding for health-care professionals as they were expected to be aware of this. The same could be said of the knowledge of the infectivity period and duration of being asymptomatic after infection. There was a good knowledge of the usage of masks among the health-care professionals.

Sixty-six percent HCWs had good attitude, while $15 \%$ and $19 \%$ had average and poor attitude, respectively, toward COVID19. Most health-care professionals expressed their distrust in the government ICMR task force on containing SARS-CoV-2 infection. Sixty-nine percent $\mathrm{HCWs}$ had good practices in preventing COVID-19 infection. Our results revealed an overall positive attitude of HCWs toward COVID-19. Good knowledge was significantly associated with this positive attitude. This is in accordance with several studies who documented that the knowledge of HCWs may be a very important prerequisite for prevention beliefs, positive attitudes, and to promote positive practices. Inadequate knowledge with other factors, as type and frequency of exposure, could increase the risk of infection [9]. Sixty-nine percent HCWs had good practices while, 31 had poor practices to combat COVID19 infection. These are in accordance to studies done by Olum et al. [11].

It is now known that the essential reproductive number (R0) of coronavirus is more in health-care professionals as compared to public and hence the relative indifference approach of healthcare professionals toward getting infected by SARS-CoV-2 is 
a concern. Therefore it is essential to know the proper use and disposal of personal protective equipment in clinical practice. In our study, 58\% HCWs knew how to correctly dispose used gloves, masks and PPE; while, $43 \%$ knew the proper donning and doffing techniques. Only $34 \%$ knew how to check whether N95 masks has been worn correctly while 56\% were aware about the correct chemical used for disinfection of COVID-19 area. In a recent study in Mumbai, $79 \%$ of the health-care professionals were aware of the various PPE required with only $54.5 \%$ of them being aware of isolation procedures needed for SARS-CoV-2 infected patients [12]. As per this data, it is highly likely that many health-care professionals will not use appropriate PPE, will get infected and further spread infections to patients [13-15].

In the present study, the questionnaire was developed based on the information available on WHO, CDC, and NHS websites and was further validated to increase the reliability of the results. Although, the study highlights the facts about the knowledge, attitude, and practices about COVID-19, has few limitations which should not be ignored. As this was a cross-sectional survey, there are chances of recall bias in information as well as it is possible that the participants may have looked up the answers to some of the questions or even discussed the answers. Despite these limitations, our findings provide valuable information about the knowledge attitude and practices of HCWs during a peak period of the pandemic.

\section{CONCLUSION}

The present study shows that the health-care professionals were quite well informed about the coronavirus. Most of the information was procured through social media. Therefore, the medical fraternity and government need to develop strategies to ensure that accurate information needs to spread in these fora. Although the study showed good knowledge among HCWs to combat Covid19 pandemic, practices and attitude needs improvement. Therefore, continued professional education is suggested among HCWs in our hospital to enhance the knowledge of HCWs to avert negative attitudes and to promote positive preventive and therapeutic practices. We recommend follow-up studies from time to time among HCWs and other staff of our hospital for ascertaining that proper knowledge is being imparted and perceived correctly.

\section{REFERENCES}

1. Bhagavathula AS, Aldhaleei WA, Rahmani J, et al. Novel Coronavirus (COVID-19) Knowledge and Perceptions: A Survey on Healthcare Workers. New York: medRxiv; 2020.
2. Wang D, Hu B, Hu C, et al. Clinical characteristics of 138 hospitalized patients with 2019 novel coronavirus-infected pneumonia in Wuhan, China. JAMA 2020;323:1061-9.

3. Wong JE, Leo YS, Tan CC. COVID-19 in Singapore-current experience: Critical global issues that require attention and action. JAMA 2020;323:1243-4.

4. Brooks SK, Dunn R, Amlôt R, et al. A systematic, thematic review of social and occupational factors associated with psychological outcomes in healthcare employees during an infectious disease outbreak. J Occup Environ Med 2018;60:248-57.

5. World Health Organization. Emerging Respiratory Viruses, Including COVID-19: Methods for Detection, Prevention, Response and Control. Geneva: World Health Organization; 2020. Available from: http://www. openwho.org/courses/introduction-to-ncov. [Last accessed on 2020 Mar 18].

6. Zhong BL, Luo W, Li HM, et al. Knowledge, attitudes, and practices towards COVID-19 among Chinese residents during the rapid rise period of the COVID-19 outbreak: A quick online cross-sectional survey. Int J Biol Sci 2020;16:1745-52.

7. Goni MD, Naing NN, Hasan H, et al. Development and validation of knowledge, attitude and practice questionnaire for prevention of respiratory tract infections among Malaysian Hajj pilgrims. BMC Public Health 2020;20:189.

8. Clements JM. Knowledge and behaviors toward COVID-19 among US residents during the early days of the pandemic: Cross-sectional online questionnaire. JMIR Public Health Surveill 2020;6:e19161.

9. Saqlain M, Munir MM, Rehman SU. Knowledge, attitude, practice and perceived barriers among healthcare workers regarding COVID-19: A crosssectional survey from Pakistan. J Hosp Infect 2020;105:419-23.

10. Abdel Wahed WY, Hefzy EM, Ahmed MI, et al. Assessment of knowledge, attitudes, and perception of health care workers regarding COVID-19, a cross-sectional study from Egypt. J Community Health 2020;45:1242-51.

11. Olum R, Chekwech G, Wekha G, et al. Coronavirus disease-2019: Knowledge, attitude, and practices of health care workers at Makerere University Teaching Hospitals, Uganda. Front Public Health 2020;8:181.

12. Modi PD, Nair G, Uppe A, et al. COVID-19 awareness among healthcare students and professionals in Mumbai metropolitan region: A questionnairebased survey. Cureus 2020;12:e7514.

13. Coronavirus Testing Kits: India to buy PPEs, but no Testing Kits, from China - The Economic Times. Available from: https://www.economictimes. indiatimes.com/news/politics-and-nation/india-to-buy-ppes-but-no-testingkits-from-china/article. [Last accessed on 2020 Apr 10].

14. Lack of PPE, Poor Infection Control Put Medical Staff at Risk of Covid-19. Available from: https://www.hindustantimes.com/india-news/lack-ofppe-poor-infection-control-put-medical-staff-at-risk-of-covid-19/st. [Last accessed on 2020 Apr 11].

15. Covid-19 Outbreak: Protective Health Gear in Short Supply - The Economic Times. Available from: https://www.economictimes.indiatimes.com/news/ politics-and-nation/covid-19-outbreak-protective-health-gear-in-shortsuppl. [Last accessed on 2020 Apr 10].

Funding: None; Conflict of Interest: None Stated.

How to cite this article: Bhasin A, Loomba P, Sharma A, Mishra B, Reshi S. Knowledge, attitude, and practices of the healthcare workers regarding 2019 novel coronavirus disease - A questionnaire study. Eastern J Med Sci. 2021;6(1):13-17.

Doi: 10.32677/EJMS.2021.v06.i01.003 